\title{
Does market exclusivity hinder the development of Follow-on Orphan Medicinal Products in Europe?
}

\author{
Anne EM Brabers ${ }^{1 *}$, Ellen HM Moors ${ }^{2}$, Sonja van Weely ${ }^{3}$ and Remco LA de Vrueh ${ }^{3}$
}

\begin{abstract}
Background: We determined whether the market exclusivity incentive of the European Orphan Drug Regulation results in a market monopoly or that absence of another Orphan Medicinal Product (OMP) for the same rare disorder, a so-called follow-on OMP, is a matter of time or market size. In the interest of rare disorder patients better understanding of the effect of the market exclusivity incentive on follow-on OMP development is warranted.

Methods: First, the impact of various market-, product- and disease-related characteristics on follow-on OMP development in the EU was determined by comparing rare disorders with an approved OMP and at least one follow-on OMP $(N=26)$, with rare disorders with an approved OMP and no follow-on OMP $(N=18)$. Next, we determined whether manufacturers continued development of a follow-on OMP upon approval of the first OMP for the intended rare disorder. Since in the EU significant benefit of an OMP has to be established, we determined for each follow-on OMP for which development was continued on what grounds significant benefit was assumed by the sponsor. Data were collected from the public domain only.
\end{abstract}

Results: The likelihood of a rare disorder with an approved OMP to obtain at least one follow-on OMP development was strongly associated with disease prevalence, turnover of the first OMP, disease class, diseasespecific scientific output and age of onset. Out of a total of 120 follow-on OMPs only one follow-on OMP could be identified for which development was discontinued upon approval of the first OMP for the same rare disorder. Only a substantial level of discontinuation of follow-on OMP development would have indicated the existence of a market monopoly. Moreover, sponsors that continued development of a follow-on OMP predominantly assumed that their product had an improved efficacy compared to the first approved OMP.

Conclusions: This study provides evidence that absence of follow-on OMP development is a matter of time or market size, rather than that the market exclusivity incentive of the European Orphan Drug Regulation creates a market monopoly.

\section{Background}

The current estimate of number of rare disorders is 6,000 to 8,000 , many of which are of genetic origin, and affect children at a very early age. In the United States (US), a rare disease is defined as a disease that affects less than 200,000 inhabitants [1]. In the EU, besides affecting fewer than five per 10,000 inhabitants, a rare disease needs to be life-threatening or chronically debilitating [1]. The total number of patients in Europe and

\footnotetext{
* Correspondence: a.brabers@nivel.nl

'NIVEL, Netherlands Institute for Health Services Research, PO Box 1568, 3500 BN Utrecht, the Netherlands

Full list of author information is available at the end of the article
}

the US suffering from a rare disorder is estimated at 55 million, and as such rare disorders are considered a real health issue $[2,3]$. Because of the rarity, the cost of developing and marketing a medicinal product to diagnose, treat or prevent a rare condition would not be recovered by the expected sales of the medicinal product under normal market conditions. Therefore, in several jurisdictions specific legislation has been introduced to stimulate the development of drugs for rare diseases, socalled orphan drugs $[4,5]$.

Since the European Union (EU) Regulation on Orphan Medicinal Products (OMP) came into force in 2000, more than 60 Orphan Medicinal Products (OMPs) have

\section{Biomed Central}


been approved for marketing and more than 830 medicinal products have received an orphan designation (OD) in the EU within the first decade of this regulation [6,7]. Just like the United States Orphan Drug Act [8-10], the EU Orphan Drug Regulation is highly appreciated for its role in creating a favorable orphan drug development environment $[10,11]$. However, this apparent success of the legislations has also been questioned. Joppi et al. argued that the large number of designated orphan drugs may provide false hope to patients with a rare disorder, because only a small percentage of these designated orphan drugs have obtained marketing approval in the EU $[12,13]$. Moreover, both in the EU and US, certain disease classes - in particular oncology - are associated with a high number of orphan designations and approvals $[9,14,15]$. In brief, the development of orphan drugs is stimulated through a number of regulatory and economic incentives, of which a market exclusivity period of seven and ten years in the US and the EU, respectively, is regarded the foremost one $[1,14]$.

Recently, Roos et al. claimed that the highly praised market exclusivity incentive basically creates a market monopoly that in their view has allowed manufacturers to charge 'exorbitant' prices for orphan drugs [16]. Tambuyzer in contrast argued that "if an approved OMP is currently the only product on the market, it is either because a company was the first to develop a treatment for this disease and competitors have yet to enter the market or because the market is too small to attract competition, rather than because the incentives have created a monopoly" [17]. To our knowledge the hypothesis that the market exclusivity incentive serves as a disincentive for the development and marketing of another OMP, a so-called follow-on OMP, next to the first approved OMP has never been tested before.

Review of the EU Community Register for Orphan Medicinal Products revealed that for several rare disorders with an approved OMP no other OMPs have been approved or are currently under development [6]. However, it also revealed that for some other rare disorders in the EU one or more OMPs have been approved next to the first OMP. For example, sildenafil, sitaxentan, iloprost and ambrisentan have all been approved as treatment for pulmonary arterial hypertension next to bosentan, the first OMP [6]. In the EU it is allowed to approve a follow-on OMP next to the first OMP, provided that significant benefit to those affected by the condition can be established. According to Commission Regulation (EC) No 847/2000 [18], significant benefit means a clinically relevant advantage or a major contribution to patient care, and is generally justified by "demonstration of potentially greater efficacy, an improved safety profile and/or more favorable pharmacokinetic properties than existing methods" [19].
Taking the aforementioned into account it remains unclear whether the market exclusivity incentive serves as a disincentive for follow-on OMP development or not. Therefore, the objective of this study was to determine whether the market exclusivity incentive of the EU Orphan Drug Regulation results in a market monopoly as argued by Roos et al. [16], or that absence of a follow-on OMP for a rare disorder for which already an approved OMP exists in the EU is a matter of time or market size as claimed by Tambuyzer [17]. First, the impact of various market-, product- and disease-related characteristics on follow-on OMP development was determined by comparing rare disorders with an approved OMP and at least one follow-on OMP, with rare disorders with an approved OMP and no follow-on OMP. Next, we determined whether manufacturers continued or discontinued development of a follow-on OMP upon approval of the first OMP for the intended rare disorder. The presence of considerable discontinuation of follow-on OMP development would suggest the existence of a market monopoly. Finally, since in the EU significant benefit of an OMP has to be established, we also determined for each follow-on OMP for which development was continued on what grounds significant benefit was assumed by the sponsor.

\section{Methods}

Characterization of rare disorders with an approved OMP

The study included all rare disorders that obtained at least one approved OMP for the treatment of that rare disorder in the EU between the start of the EU Regulation on Orphan Medicinal Products (April 2000) and 31 December 2008. The study was limited to rare disorders with an approved treatment, since only one rare disorder with an approved OMP for the diagnosis or prevention of that disorder could be identified. For each rare disorder with an approved OMP the EU register of Orphan Medicinal Products was checked for follow-on OMPs between the start of the EU Regulation on Orphan Medicinal Products (April 2000) and 30 April 2010. The reason for extending the cut-off date was to maximize detection of follow-on OMPs. A follow-on OMP was defined as: (a) another approved OMP for the same rare disorder in Europe (e.g. both agalsidase bèta and agalsidase alfa are approved for the treatment of Fabry disease in Europe) or (b) another medicinal product with a European orphan designation (OD) for the same disorder. Only OMPs that were under development at or after marketing approval of the first OMP for the same rare disorder were identified as followon OMP and included in the study. An OMP designated before approval of the first OMP for the same rare disorder, but whose development was discontinued before approval of the first OMP was not considered as follow-on OMP. Finally, rare disorders included in the study were 
divided into two groups: (a) rare disorders having an approved OMP and at least one follow-on OMP ('Followon' group), and (b) rare disorders having an approved OMP, but no follow-on OMP ('no Follow-on' group).

\section{Factors elucidating follow-on OMP development}

Data were collected for each rare disorder on product-, market- and disease-related characteristics to get insight in factors elucidating follow-on OMP development. Selection of data was limited to the public domain and essentially similar to Heemstra et al. [20,21].

Market-related characteristics included data on the prevalence of the rare disorder, the annual turnover of the first OMP, and whether the first OMP was designated or approved outside Europe. Product-related characteristics included data on the pharmaceutical formulation. Disease-related characteristics included data on the disease class, the disease-specific scientific output (i.e. the number of scientific publications) and whether the rare disorder was a childhood, chronic or inheritable disorder [20,21]. A full list of these characteristics, definitions and the (main) public sources used, is depicted in table 1 .

The characteristics of rare disorders having at least one follow-on OMP ('Follow-on' group) and rare disorders with no follow-on OMP ('no Follow-on' group) were compared using univariate analyses. Odds ratios (OR) and 95\% confidence intervals (CI) were calculated for each characteristic. All analyses were done using SPSS, version 16.0.

\section{Effect of marketing authorization of first OMP on follow- on OMP development}

Upon marketing approval of a first OMP, sponsors of a follow-on OMP for the same rare disorder could either decide to continue or discontinue development. In this study a sponsor continued development if: (a) the orphan designation date of the follow-on OMP, based on the EU orphan drug register, was after the date of marketing authorization of the first OMP or (b) if continuation of development was verified in the public domain for follow-on OMPs having a designation date prior to the approval date of the first OMP. As a result, the following two groups were discerned: (a) sponsors that continued development of a follow-on OMP upon marketing approval of the first OMP and (b) sponsors that discontinued development of a follow-on OMP upon marketing approval of the first OMP.

\section{Characterization of assumptions of significant benefit of follow-on OMPs}

The European Medicines Agency (EMA) guideline COMP/15893/2009 outlines the necessary level of evidence to support assumptions of significant benefit [19].
Significant benefit is justified in the EU by demonstrating an improved efficacy, a better safety or a major contribution to patient care. In this study the concept of significant benefit included these three categories. Data regarding the assumptions of significant benefit were collected using sources in the public domain. Firstly, European Public Assessment Reports (EPARs) were consulted for follow-on OMPs that were successfully developed and approved in the EU. An EPAR provides information regarding the significant benefit of a followon OMP compared to earlier approved OMPs for the same rare disorder. Secondly, Summaries of Opinion were consulted provided that the follow-on OMP obtained an orphan designation after approval of the first OMP for the same rare disorder. Finally, sponsor documents (e.g. sponsor website, annual reports, press releases) were consulted to determine the assumptions of significant benefit of follow-on OMPs that obtained an orphan designation before approval of the first OMP for the same rare disorder. All collected assumptions of significant benefit were compared and mapped using central key words of the mentioned EMA guideline [19] for each of the three categories (for example mechanism of action for improved efficacy, side effects for better safety and way of administration for a major contribution to patient care).

\section{Results}

Characterization of rare disorders with an approved OMP From the start of the EU Regulation on Orphan Medicinal Products (April 2000) up to 31 December 2008, 58 OMPs intended for the treatment of a rare disorder obtained marketing approval in the EU [6]. Although some products (e.g. imatinib and dasatinib) have been approved for more than one rare disorder, most approved OMPs are intended as treatment for only one specific rare disorder. Collectively, the 58 approved OMPs have been authorized as treatments for 44 different rare disorders. Of these 44 rare disorders, 26 had at least one follow-on OMP ('Follow-on' group) and 18 did not have a follow-on OMP ('no Follow-on' group), as reflected in table 2 .

A review of the EU Community Register of Orphan Medicinal Products revealed that the 26 rare disorders in the 'Follow-on' group encompass a total of 163 orphan designations (ODs) between the start of the EU Orphan Drug Regulation (April 2000) and 30 April 2010 in the EU [6]. Analysis of the 163 ODs showed that development of 13 orphan drugs had been discontinued before marketing approval of the first OMP for the same rare disorder. In addition, one orphan drug was intended for the diagnosis rather than for the treatment of a rare disorder. From the remaining 149 ODs, 120 orphan drugs were identified as being under 
Table 1 Definitions and sources used for market-, product- and disease-related characteristics of Orphan Medicinal Products (OMPs)

\begin{tabular}{|c|c|c|}
\hline Characteristic & Definitions & (Main) public source \\
\hline \multicolumn{3}{|l|}{ Market-related characteristics } \\
\hline prevalence & $\begin{array}{l}<1 \text { per } 100,000 \\
\text { between } 1-50 \text { per 100,000 }\end{array}$ & Orpha.net (report series, Nov 2009) \\
\hline annual turnover first OMP & maximum annual turnover of the first OMP & Sponsor documents \\
\hline \multirow[t]{2}{*}{ first OMP designated outside EU } & first OMP obtained an orphan designation & FDA Orphan Drug \\
\hline & inside and outside Europe & Product database \\
\hline \multirow[t]{2}{*}{ first OMP authorized outside EU } & first OMP obtained marketing authorization & FDA Orphan Drug \\
\hline & inside and outside Europe & Product database \\
\hline \multicolumn{3}{|l|}{ Product-related characteristics } \\
\hline pharmaceutical formulation & $\begin{array}{l}\text { parenteral } \\
\text { oral }\end{array}$ & EPARs \\
\hline \multicolumn{3}{|l|}{ Disease-related characteristics } \\
\hline disease class (ICD-10) & $\begin{array}{l}\text { other ICD-10 classes } \\
\text { C00-D48 (oncologic disorders) }\end{array}$ & Orpha.net \\
\hline disease-specific scientific output & number of scientific publications in the period $1976-2008$ in PubMed for a specific rare disorder & PubMed \\
\hline inheritable disease & majority of cases caused by genetic inheritance & Orpha.net \\
\hline chronic disease & disease duration is generally over 3 months & Orpha.net \\
\hline childhood disease & majority of diagnosis before age 18 & Orpha.net \\
\hline
\end{tabular}


Table 2 Rare disorders with an approved OMP in EU until 31 December 2008

Rare disorder
$(\mathrm{N}=44)$

Fabry disease

Chronic myeloid leukemia

Gaucher disease

Acute promyelocytic leukemia

Pulmonary arterial hypertension

Acromegaly

Gastrointestinal stromal tumors

Familial adenomatous polypopsis

NAGS-deficiency

Treatment prior to hematopoietic progenitor cell

transplantation

Mucopolysaccharidosis type I

Essential thrombocythaemia

Wilson's disease

High-grade dysplasia in Barrett's oesophagus

Patent ductus arteriosus

Adrenal cortical carcinoma

Hairy cell leukemia

Narcolepsy-cataplexy

Chronic pain requiring intraspinal analgesia

Tyrosinaemia type I (hereditary)

Dermatofibrosarcoma protuberans

Acute lymphoblastic leukemia

Anthracycline extravasations

Renal cell carcinoma

Mucopolysaccharidosis type VI

Glycogen storage disease type ॥

Chronic iron overload requiring chelation therapy

Hypereosinophilic syndrome

Myelodysplastic syndromes

Hepatocellular carcinoma

Soft tissue sarcoma

Paroxysmal nocturnal haemoglobinuria

Sickle cell syndrome

Multiple myeloma

Lennox-Gastaut syndrome

Severe primary IGF-1 deficiency

Mucopolysaccharidosis type ॥

Severe myoclonic epilepsy in infancy (Dravet's syndrome)

Homocystinuria

Systemic sclerosis

Chronic myelomonocytic leukemia

Hyperphenylalaninaemia

Angioedema (hereditary)

Acute myeloid leukemia

First OMP
(INN-name)

Other ODs*

agalsidase alfa/agalsidase beta**

$(\mathrm{N}=149)$

imatinib

miglustat

arsenic trioxide

bosentan

pegvisomant

imatinib

celecoxib

carglumic acid

busulfan

laronidase

anagrelide

zinc acetate dihydrate

porfimer sodium

ibuprofen

mitotane

cladribine

sodium oxybate

ziconotide

nitisinone

imatinib

clofarabine

dexrazoxane

sorafenib

galsulfase

alglucosidase alpha

deferasirox

imatinib

imatinib

sorafenib

trabectedin

eculizumab

hydroxycarbamide 3

lenalidomide 14

rufinamide

mecasermin

idursulfase

stiripentol

betaine anhydrous

bosentan

azacitidine

sapropterin dihydrochloride

icatibant

histamine dihydrochloride
7

3

2

2

3

2

$-$

$-$

$-$

2

3

27

* Other ODs in the EU under development for treatment of the specific rare disorder between the start of the EU Orphan Drug Regulation (April 2000) and 30 April 2010.

** Both agalsidase alfa and agalsidase beta obtained market approval at the 3th of August 2001 in the EU. 
development at or after approval of the first OMP and included as follow-on OMP in this study. Despite an in depth search into multiple sources in the public domain, for the remaining 29 ODs we were unable to determine whether these orphan drugs were still under development at or after approval of the first OMP for the same rare disorder. Consequently, these ODs could not be identified as follow-on OMPs and were not included in the study.

\section{Factors elucidating follow-on OMP development}

The results of the univariate comparisons between rare disorders with an approved OMP and at least one follow-on OMP $(n=26)$ and rare disorders with an approved OMP and no follow-on OMP $(\mathrm{n}=18)$ are shown in table 3 . For some of the characteristics statistically significant relations were observed, however, the association was strongest for the scientific output of a specific disorder. A rare disorder with more than 450 scientific publications in PubMed in the period 19762008 was associated with a 25 -fold increase in the chance to obtain at least one follow-on OMP compared to a rare disorder with less than 450 scientific publications in this period $(\mathrm{OR}=25.0 ; \mathrm{CI}=2.8-226.1)$. Furthermore, the results showed that oncologic disorders (ICD10 class C00-D48) with an approved OMP have an 8fold increased likelihood to obtain at least one follow-on OMP compared to rare disorders in other ICD-10 classes $(\mathrm{OR}=8.0 ; \mathrm{CI}=1.5-42.0)$. A statistically significant relation was also found for the annual turnover of the first approved OMP. Rare disorders having a first approved OMP with more than 50 million US dollar annual sales have an almost 5-fold higher probability to obtain a follow-on OMP than rare disorders having a first approved OMP with annual sales below 50 million US dollar $(\mathrm{OR}=4.8 ; \mathrm{CI}=1.1-21.4)$. Finally, a striking result is that rare disorders with an age of onset in childhood have a 5-fold lower chance to obtain at least one follow-on OMP than rare disorders with an age of onset in adulthood $(\mathrm{OR}=0.2 ; \mathrm{CI}=0.1-0.8)$.

The descriptive statistics showed a strong association between the disease prevalence and the likelihood to obtain at least one follow-on OMP. All rare disorders with a first approved OMP and at least one follow-on OMP had a prevalence between $1 / 100,000$ and 50/ 100,000 , whereas rare disorders with a first approved OMP and no follow-on OMP were more or less equally divided over both prevalence categories $(<1 / 100,000$ and $1-50 / 100,000)$.

\section{Effect of marketing authorization of first OMP on follow- on OMP development}

To elucidate whether the instrument of market exclusivity served as disincentive, we determined the effect of marketing authorization of a first OMP on follow-on development. Overall, our results show that development of follow-on OMPs is continued after marketing approval of a first OMP for the same rare disorder. Out of a total of 120 follow-on OMPs only one follow-on OMP, PI-88 of the Australian company Progen, could be identified for which development was discontinued. According to the company the decision of discontinuation "was made based on a number of factors, but was primarily driven by the competitive pressures in Europe and North America of Bayer's launching a Nexavar ${ }^{\circledR}$ trial amongst the same patients" [22].

\section{Characterization of assumptions of significant benefit of follow-on OMPs}

Assumptions of significant benefit were found for 106 of the 119 follow-on OMPs in the EU. For the remaining 13 follow-on OMPs no assumptions of significant benefit could be identified, predominantly due to lack of information in sponsor documents for follow-on OMPs that obtained an orphan designation (OD) before marketing approval of the first OMP $(n=10)$. For the other three follow-on OMPs the Summary of Opinion did not provide clear information regarding the assumption of significant benefit (e.g. "the product might be of potential significant benefit"). Consequently, these follow-on OMPs were not included in the study.

For several follow-on OMPs $(n=22)$ more than one assumption of significant benefit was found, resulting in a total of 130 different assumptions, as depicted in table 4. Most assumptions of significant benefit (85.5\%; $\mathrm{n}=$ 111) were related to the concept of improved efficacy. Within this category, most sponsors assumed that their follow-on OMP would be of significant benefit due to a different mechanism of action, followed by treatment of a subgroup of patients. The assumptions of significant benefit for the remaining 19 follow-on OMPs were related to an improved safety profile $(n=9)$ or a major contribution to patient care $(\mathrm{n}=10)$ in comparison with the first OMP for the same rare disorder, respectively. More than half of the follow-on OMPs in the category of improved safety were assumed to have fewer side effects compared to existing treatments. With regard to the contribution to patient care it was observed that, except for one follow-on OMP, all assumptions were related to the mode of administration.

\section{Discussion}

In the interest of patients with a rare disorder, but also of other stakeholders, better understanding of the effect of the market exclusivity incentive on follow-on OMP development for a rare disorder for which an approved OMP exists is warranted. The objective of this study was to determine whether the market exclusivity 
Table 3 Comparison between rare disorders with follow-on and no follow-on OMPs: Descriptive statistics and univariate analyses

\begin{tabular}{|c|c|c|c|c|c|}
\hline \multirow[t]{2}{*}{ Characteristic } & \multirow[t]{2}{*}{ Indicator } & \multicolumn{3}{|c|}{ Rare disorders } & \multirow[b]{2}{*}{ Odds Ratio ( $95 \%$ Confidence Interval } \\
\hline & & $\begin{array}{l}\text { Total } \\
\mathrm{N}=44\end{array}$ & $\begin{array}{l}\text { 'Follow-on' Group } \\
\mathrm{N}=26\end{array}$ & $\begin{array}{l}\text { 'No Follow-on' Group } \\
N=18\end{array}$ & \\
\hline \multicolumn{6}{|l|}{ Market-related } \\
\hline \multirow[t]{2}{*}{ prevalence } & $<1$ per 100,000 & 9 & $0^{*}$ & 9 & reference level \\
\hline & between 1 - 50 per 100,000 & 34 & 26 & 8 & not applicable \\
\hline turnover first & $<50$ mil. US \$ & 11 & 4 & 7 & reference level \\
\hline OMP & $>50$ mil. US \$ & 26 & 19 & 7 & $4.8(1.1-21.4)$ \\
\hline designated outside & no & 8 & 5 & 3 & reference level \\
\hline EU & yes & 36 & 21 & 15 & $0.8(0.2-4.1)$ \\
\hline approved outside & no & 11 & 7 & 4 & reference level \\
\hline EU & yes & 33 & 19 & 14 & $0.8(0.2-3.2)$ \\
\hline \multicolumn{6}{|l|}{ Product-related } \\
\hline pharmaceutical & parenteral & 20 & 9 & 11 & reference level \\
\hline formulation & oral & 24 & 17 & 7 & $3.0(0.9-10.3)$ \\
\hline \multicolumn{6}{|l|}{ Disease-related } \\
\hline \multirow[t]{2}{*}{ disease class } & other ICD-10 classes & 29 & 13 & 16 & reference level \\
\hline & C00-D48 & 15 & 13 & 2 & $8.0(1.5-42.0)$ \\
\hline disease-specific & $<450$ publications & 10 & 1 & 9 & reference level \\
\hline scientific output & > 450 publications & 34 & 25 & 9 & $25.0(2.8-226.1)$ \\
\hline \multirow[t]{2}{*}{ childhood } & adulthood & 22 & 17 & 5 & reference level \\
\hline & childhood & 17 & 7 & 10 & $0.2(0.1-0.8)$ \\
\hline \multirow[t]{2}{*}{ chronic } & non-chronic & 5 & 3 & 2 & reference level \\
\hline & chronic & 34 & 19 & 15 & $0.8(0.1-5.7)$ \\
\hline \multirow[t]{2}{*}{ inheritable } & non-inheritable & 28 & 18 & 10 & reference level \\
\hline & inheritable & 16 & 8 & 8 & $0.6(0.2-1.9)$ \\
\hline
\end{tabular}

* Zero cell count/no follow-on OMP 
Table 4 Assumptions of significant benefit of the follow-on OMPs for which development was continued

\begin{tabular}{|c|c|c|c|c|}
\hline \multirow[t]{2}{*}{ Category of significant benefit } & \multirow{2}{*}{\multicolumn{2}{|c|}{ Classes of significant benefit }} & \multicolumn{2}{|c|}{ Assumptions } \\
\hline & & & $N=130$ & $\%$ \\
\hline \multirow[t]{8}{*}{ Improved efficacy } & Different mechanism of action & & 47 & 36.2 \\
\hline & Sub-group (including patients who do not respond & & & \\
\hline & on current treatments) & & 27 & 20.8 \\
\hline & Alternative/additional treatment & & 14 & 10.8 \\
\hline & Improve the long-term outcome of the patient & & 12 & 9.2 \\
\hline & More effective (unspecified) & & 10 & 7.7 \\
\hline & Improve the treatment (unspecified) & & 1 & 0.8 \\
\hline & & Total & 111 & 85.5 \\
\hline \multirow[t]{4}{*}{ Improved safety } & Fewer side effects & & 5 & 3.8 \\
\hline & Increased tolerability & & 2 & 1.5 \\
\hline & Improved safety (unspecified) & & 2 & 1.5 \\
\hline & & Total & 9 & 6.8 \\
\hline \multirow[t]{3}{*}{ Contribution to patient care } & Mode of administration & & 9 & 6.9 \\
\hline & Wider availability & & 1 & 0.8 \\
\hline & & Total & 10 & 7.7 \\
\hline
\end{tabular}

incentive of the EU Orphan Drug Regulation results in a market monopoly, as argued by Roos et al. [16], or that absence of follow-on OMPs is a matter of time or market size, as claimed by Tambuyzer [17]. First, the impact of various market-, product- and disease-related characteristics on follow-on OMP development in the EU was determined. Comparison of rare disorders with a first approved OMP and at least one follow-on OMP ('Follow-on' group) with rare disorders with a first approved OMP and no follow-on OMP ('no Follow-on group') revealed disease prevalence, turnover of the first OMP, disease class, disease-specific scientific output and age of onset as predictors of follow-on OMP development. Although it was not possible to perform a multivariate analysis and to test whether any of the aforementioned characteristics were mutually related, it is suspected that some level of mutual relationship may exist. Heemstra et al. showed that scientific output for oncologic rare disorders in general is higher than for other rare disorders, which suggests some degree of mutual relationship between disease class and scientific output [20]. Also, the prevalence of a rare disorder and turnover of the first OMP may to some extent be mutually related. Despite the possibility of mutual relationships, our findings are in line with several studies that focused on characteristics that play a driving role in the orphan drug development process [20,23-25].

The lower likelihood on follow-on OMP development for rare childhood disorders is most likely a natural consequence of the general reluctance of the pharmaceutical industry to invest in the development of specific therapies for a population that is small in number and fragmented into several age groups with often unique requirements $[26,27]$. To stimulate paediatric drug development in the EU Regulation (EC) No 1901/2006 on medicinal products for paediatric use came into force in January 2007 [28]. Through a number of regulatory and economic incentives, including extension of the market exclusivity period from ten to twelve years for OMPs intended for a paediatric indication, the legislation intends to have a positive impact on paediatric drug development, although risks and pitfalls remain [29]. Conducting clinical trials for rare indications, especially very rare ones, remains difficult due to a number of well-known practical limitations (e.g. lack of proper diagnosis, finding enough patients, geographical distribution of patient population) [30]. Successful drug development requires thorough disease knowledge, such as its etiology and pathophysiology [25]. The latter explains our finding that, next to initiation of an orphan drug development program [20], the level of diseasespecific scientific output increases the likelihood on follow-on OMP development. It also explains our observation that for rare oncologic diseases the likelihood on follow-on OMP development is higher than for other ICD disease classes. Considerable public and private expenditure on research and a high-level of transnational research infrastructure have resulted in oncology having the highest scientific output in the area of rare diseases [31,32]. Consequently, oncology represents an attractive indication for the pharmaceutical sector $[33,34]$, resulting in the highest number of first and follow-on OMPs in development as well as approved [6]. Because of its dynamic and divergent nature, biomedical research can result in several competing hypotheses on the possible causes of diseases, and several promising targets and therapeutical approaches [35]. This makes biomedical research an extremely complex process to 
manage, and as a result translation of disease knowledge into (follow-on) OMP development requires time.

In contrast to the claim by Roos et al. that the market exclusivity incentive basically creates a market monopoly that in their view allows manufacturers to charge 'exorbitant' prices for orphan drugs [16], we found that turnover of the first OMP in fact increases the likelihood on follow-on OMP development. The presence of an approved OMP for a rare disorder is a clear indication that development and marketing of a therapy for that specific rare disorder is attainable. However, the mere presence of an approved OMP for a rare disorder is not sufficient; a certain level of profitability is apparently an important prerequisite for the initiation or continuation of the development of a follow-on OMP.

Our finding that prevalence of a rare disease is associated with an increased likelihood on follow-on OMP development provides corroborative evidence for an element in Tambuyzer's hypothesis that if an approved OMP is currently the only product on the market, this can be explained because the market is too small to attract competition [17]. Acemoglu and Linn more generally revealed that an increase in the potential market size for a drug category correlates with an increase in the number of new drugs in that category [24]. The importance of prevalence of a rare disease, which can be regarded as a surrogate marker for market size, was already confirmed in other stages of the orphan drug development process. Heemstra et al. showed that prevalence is a predictor for the translation of rare disorder research into the initiation of an orphan drug development program [20]. Yin reported that "the US Orphan Drug Act has led to a significant and sustained increase in new trials" among more prevalent rare diseases, but not for less prevalent rare diseases [23].

Tambuyzer's hypothesis is further supported by our finding that in the EU all manufacturers of follow-on OMPs upon approval of the first OMP for the same rare disorder decided to continue development of their product, except for one. Only a substantial level of discontinuation of follow-on OMP development would have indicated the existence of a market monopoly. Discontinuation of several follow-on OMPs was found, however, reasons for discontinuation were related to conducting clinical trials, focus on another more promising indication, registration failure and lack of funding. Apparently, discontinuation of follow-on OMPs is not directly associated with market approval of a first OMP for the same rare disorder, but rather due to safety-, efficacy-, or economic-related factors similar to the majority of regular drug development discontinuation [36]. Interestingly, we found that 15 of the 120 follow-on OMPs (12.5\%) subsequently obtained a marketing authorization in the EU between the start of the legislation and April 2010. This is considerably higher than the 45 of 528 orphan designations (8.5\%) that were approved in the EU between the start of the legislation and 2007 [12]. One explanation for this observation could be that the majority of follow-on OMPs is in development by large pharmaceutical firms (data not shown), whereas most orphan designated products are being developed by Small and Medium-sized Enterprises (SMEs) [37]. In general, SMEs have less experience in (orphan) drug development, which was reported by Heemstra et al. as an important predictor for OMP approval [21].

Finally, we found that sponsors that continue development of a follow-on OMP in the EU predominantly assume that their product has an improved efficacy compared to the first OMP. Establishing the assumption of significant benefit based on an improved safety and/or major contribution to patient care may be either more difficult for or less attractive to manufacturers of followon OMPs. The EMA guideline on significant benefit mentions that "a theoretical risk with an authorized product cannot be compared with a theoretical lack of risk with the product under development" [19]. However, most applications for an orphan designation by a sponsor are done in the late preclinical or early clinical testing stage, which doesn't include extensive human safety testing. Moreover, serious adverse events with authorized orphan medicinal products have been limited until now [38]; only sitaxentan was voluntarily withdrawn from the market by Pfizer in 2010 due to new information on two cases of fatal liver injury [39]. The reason for the limited number of follow-on OMPs with significant benefit based on an assumption of a major contribution to patient care may be best explained by a current lack of interest by the pharmaceutical industry. Increased patient involvement and demand may result in shifting focus to novel innovative drug delivery systems that will allow more selective, more precise, less frequent dosing of the orphan medicinal product $[40,41]$.

\section{Conclusion}

This study provides evidence for the hypothesis that approval of the first OMP for a rare disorder, and consequently a market exclusivity period of ten years, does not serve as a disincentive for the development and marketing of a follow-on OMP for the same indication in the EU. We found that absence of follow-on OMP development is more a matter of time or market size as argued by Tambuyzer [17], rather than the creation of a market monopoly as claimed by Roos et al. [16]. Apart from market size, turnover of the first OMP, disease class, disease-specific scientific output and age of onset were identified as additional predictors of follow-on OMP development. In general, regardless of approval of 
the first OMP for the intended rare disorder, development of a follow-on OMP is continued and primarily based on the assumption of improved efficacy.

Finally, within the area of rare diseases the role of patient organizations is strong, a real added value, and continuously growing $[40,41]$. It will be interesting to monitor if this increased patient involvement and demand will result in expanding the focus of the pharmaceutical industry to novel innovative drug delivery systems that will allow more selective, more precise, less frequent dosing of the orphan medicinal product [40]. Ultimately, we believe that, next to improved efficacy, this kind of follow-on OMP development truly represents an important improvement in current care of patients with a rare disorder.

\section{Author details}

${ }^{1}$ NIVEL, Netherlands Institute for Health Services Research, PO Box 1568, 3500 BN Utrecht, the Netherlands. ${ }^{2}$ Innovation Studies, Copernicus Institute, Utrecht University, Heidelberglaan 2, 3584 CS Utrecht, the Netherlands. ${ }^{3}$ Dutch Steering Committee on Orphan Drugs, Laan van Nieuw Oost Indië 334, 2593 CE Den Haag, the Netherlands.

\section{Authors' contributions}

RDV and SWW were responsible for the study idea. AB designed and performed the study, was the primary responsible person for data collection and data evaluation. RDV, EM and SWW participated in the study design and data evaluation. RDV and EM were involved in the supervision of this study. $A B$ wrote together with RDV the manuscript and SWW and EM revised the manuscript. All authors read and approved the final manuscript.

\section{Competing interests}

The authors declare that they have no competing interests.

Received: 14 July 2011 Accepted: 5 September 2011

Published: 5 September 2011

\section{References}

1. Dear JW, Lilitkarntakul P, Webb DJ: Are rare diseases still orphans or happily adopted? The challenges of developing and using orphan medicinal products. Br J Clin Pharmacol 2006, 62:264-271.

2. Stolk P, Willemen MJ, Leufkens HG: Rare essentials: drugs for rare diseases as essential medicines. Bull World Health Organ 2006, 84(9):745-751.

3. Schieppati A, Henter Jl, Daina E, Aperia A: Why rare diseases are an important medical and social issue. Lancet 2008, 371(9629):2039-2041.

4. The Orphan Drug Act. United States Public Law No 97-414. [http://www. fda.gov/RegulatoryInformation/Legislation/

FederalFoodDrugandCosmeticActFDCAct/

SignificantAmendmentstotheFDCAct/OrphanDrugAct/default.htm]

5. Regulation (EC) No. $141 / 2000$ of the European Parliament and of the Council of 16 December 1999 on Orphan Medicinal Products. [http://eurlex.europa.eu/LexUriServ/LexUriServ.do?uri=OJ:L:2000:018:0001:0005:en:PDF].

6. European Commission Register of designated Orphan Medicinal Products. [http://ec.europa.eu/health/documents/community-register/html/ orphreg.htm]

7. The Committee for Orphan Medicinal Products and the European Medicines Agency Scientific Secretariat: European regulation on orphan medicinal products: 10 years of experience and future perspectives. Nat Rev Drug Discov 2011, 10(5):341-349.

8. Haffner ME: Adopting orphan drugs - two dozen years of treating rare diseases. N Engl J Med 2006, 354:445-447.

9. Haffner ME, Torrent-Farnell J, Maher PD: Does orphan drug legislation really answer the needs of patients? Lancet 2008, 371:2041-2044.

10. Rinaldi A: Adopting an orphan. EMBO Rep 2005, 6:507-510.
11. Committee for Orphan Medicinal Products report to the Commission in relation to article 10 of Regulation 141/2000 on orphan medicinal products. [http://www.ema.europa.eu/docs/en_GB/document_library/ Report/2010/04/WC500089638.pdf].

12. Joppi $R$, Bertele $V$, Garattini S: Orphan drug development is not taking off. Br J Clin Pharmacol 2009, 67(5):494-502.

13. Joppi R, Bertele V, Garattini S: Orphan drug development is progressing too slowly. Br J Clin Pharmacol 2006, 61(3):355-360.

14. Meyers A, Lipucci Di Paola M: The orphan medicinal products: an international challenge. Minerva Biotecnol 2003, 15:161-166.

15. Commission staff working document on the experience acquired as a result of the application of Regulation (EC) No. 141/2000 on orphan medicinal products and account of the public health benefits obtained. [http://ec.europa.eu/health/files/orphanmp/doc/orphan_en_06-2006_en.pdf].

16. Roos JC, Hyry HI, Cox TM: Orphan drug pricing may warrant a competition law investigation. BMJ 2010, 16:341.

17. Tambuyzer E: Rare diseases, orphan drugs and their regulation: questions and misconceptions. Nat Rev Drug Discov 2010, 9(12):921-929.

18. Commission Regulation (EC) No. $847 / 2000$ of 27 April 2000 laying down the provisions for implementation of the criteria for designation of a medicinal product as an orphan medicinal product and definitions of the concepts 'similar medicinal product' and 'clinical superiority'. [http:// ec.europa.eu/health/files/eudralex/vol-1/reg_2000_847/reg_2000_847_en. pdf].

19. Committee for Orphan Medicinal Products. Guideline on elements required to support the medical plausibility and the assumption of significant benefit for an orphan designation. [http://www.emea.europa. eu/docs/en_GB/document_library/Regulatory_and_procedural_guideline/ 2010/07/WC500095341.pdf].

20. Heemstra HE, van Weely S, Büller HA, Leufkens HGM, de Vrueh RL: Translation of rare disease research into orphan drug development: disease matters. Drug Discov Today 2009, 14(23-24):1166-1173.

21. Heemstra HE, de Vrueh RL, van Weely S, Büller HA, Leufkens HG: Predictors of orphan drug approval in the European Union. Eur J Clin Pharmacol 2008, 64(5):545-552.

22. Progen: Progen and Avexa agree to withdraw from merger.[http://www. progen.com.au/Docs/prs/Progen\%20withdraws\%20from\%20Avexa\% 20Merger.pdf].

23. Yin W: Market incentives and pharmaceutical innovation. $J$ Health Econ 2008, 27(4):1060-1077.

24. Acemoglu D, Linn J: Market size in innovation: theory and evidence from the pharmaceutical industry. NBER Working Papers 10038 National Bureau of Economics Research 2003, 1-67.

25. Griggs RC, Batshaw M, Dunkle M, Gopal-Srivastava R, Kaye E, Krischer J, et al: Clinial research for rare disease: opportunities, challenges, and solutions. Mol Genet Metab 2009, 96(1):20-26.

26. Rose K: Better medicines for children - where are we now, and where do we want to be? Br J Clin Pharmacol 2005, 59(6):657-659.

27. Saint Raymond A, Brasseur D: Development of medicines for children in Europe: ethical implications. Paediatr Respir Rev 2005, 6(1):45-51.

28. Regulation (EC) No. 1901/2006 of the European Parliament and of the Council of 12 December 2006 on medicinal products for paediatric use and amending Regulation (EEC) No. 1768/92, Directive 2001/20/EC, Directive 2001/83/EC and Regulation (EC) No 726/2004. [http://ec.europa. eu/health/files/eudralex/vol-1/reg_2006_1901/reg_2006_1901_en.pdf].

29. Rocchi F, Paolucci P, Ceci A, Rossi P: The European paediatric legislation: benefits and perspectives. Ital J Pediatr 2010, 36:56.

30. Wästfelt M, Fadeel B, Henter J: A journey of hope: lessons learned from studies on rare diseases and orphan drugs. J Intern Med 2006, 260(1):1-10

31. Eckhouse S, Lewison G, Sullivan R: Investments and outputs of cancer research: from the public sector to industry.[http://www. ecancermedicalscience.com/cache/pdf/ECRM\%20LR\%20Survey.pdf].

32. Eckhouse S, Sullivan R: A survey of public funding of cancer research in the European union. PLoS Med 2006, 3(7):e267.

33. Schmid EF, Smith DA: Keynote review: Is declining innovation in the pharmaceutical industry a myth? Drug Discov Today 2005, 10(15):1031-1039.

34. Stratton MR, Campbell PJ, Futreal PA: The cancer genome. Nature 2009, 458:719-724.

35. Bonaccorsi A: Search regimes and the industrial dynamics of science. Minerva 2008, 46(3):285-315. 
36. DiMasi JA: Risks in new drug development: approval success rates for investigational drugs. Clin Pharmacol Ther 2001, 69(5):2997-307.

37. Torrent-Farnell J: Five years with the Orphan Drug Directive in the EU Achievements by the COMP.[http://icord.se/past-events/stockholm_2005/ speakers_presentations].

38. Heemstra HE, Giezen TJ, Mantel-Teeuwisse AK, de Vrueh RL, Leufkens HG: Safety-related regulatory actions for orphan drugs in the US and EU: a cohort study. Drug Saf 2010, 33(2):127-137.

39. European Medicines Agency: Thelin (sitaxentan) to be withdrawn due to cases of unpredictable serious liver injury.[http://www.ema.europa.eu/ ema/index.jsp?curl=pages/news_and_events/news/2010/12/

news_detail_001161.jsp\&murl=menus/news_and_events/news_and_events. jsp\&mid=WC0b01ac058004d5c1\&jsenabled=true ].

40. Boon WPC, Moors EHM, Kuhlmann S, Smits REHM: Demand articulation in intermediary organisations: the case of orphan drugs in the Netherlands. Technological Forecasting and Social Change 2008, 75(5):644-671

41. Aymé S, Kole A, Groft S: Empowerment of patients: lessons from the rare diseases community. Lancet 2008, 371(9629):2048-2051.

doi:10.1186/1750-1172-6-59

Cite this article as: Brabers et al: Does market exclusivity hinder the development of Follow-on Orphan Medicinal Products in Europe?

Orphanet Journal of Rare Diseases 2011 6:59.

\section{Submit your next manuscript to BioMed Central} and take full advantage of:

- Convenient online submission

- Thorough peer review

- No space constraints or color figure charges

- Immediate publication on acceptance

- Inclusion in PubMed, CAS, Scopus and Google Scholar

- Research which is freely available for redistribution

Submit your manuscript at www.biomedcentral.com/submit 